\title{
K-cluster-valued compressive sensing for imaging
}

\author{
Mai Xu ${ }^{*}$ and Jianhua Lu
}

\begin{abstract}
The success of compressive sensing (CS) implies that an image can be compressed directly into acquisition with the measurement number over the whole image less than pixel number of the image. In this paper, we extend the existing CS by including the prior knowledge of K-cluster values available for the pixels or wavelet coefficients of an image. In order to model such prior knowledge, we propose in this paper K-cluster-valued CS approach for imaging, by incorporating the $K$-means algorithm in CoSaMP recovery algorithm. One significant advantage of the proposed approach, rather than the conventional CS, is the capability of reducing measurement numbers required for the accurate image reconstruction. Finally, the performance of conventional CS and K-cluster-valued CS is evaluated using some natural images and background subtraction images.
\end{abstract}

Keywords: compressive sensing, K-means algorithm, model-based method

\section{Introduction}

Image compression is currently an active research area, as it offers the promise of making the storage or transmission of images more efficient. The aim of image compression [1] is to reduce the data size of image and then make the image stored or transmitted in an efficient form. In image compression, we may transform the image into an appropriate basis and only store or transmit the important expansion coefficients [2]. Since such coefficients are normally sparse (only few coefficients are nonzero) or compressible (decaying rapidly according to power law), the compression (e.g., image compression in JEPG2000 [3]) can be achieved via storing and transmitting the nonzero coefficients.

For example, assume that we have acquired an image signal $\boldsymbol{x} \in \mathbb{R}^{N}$ with $N$ pixels. Through DCT or wavelet transform, image $x$ may be represented in terms of sets of coefficients via a basis expansion: $\boldsymbol{x}=\boldsymbol{\Psi} \boldsymbol{\alpha}$, where $\boldsymbol{\Psi}$ is an $N \times N$ basis matrix. Therefore, $x$ may be represented by sparse coefficients $\boldsymbol{\alpha}=\left\{\alpha_{i}\right\}_{1}^{N}$, where $S(\ll N)$ coefficients are nonzero and then only these $S$ coefficients with their locations need to be stored such that the compression can be achieved. Note that such $\alpha$ is defined as $S$-sparse. In practice, it is clear [4] that the natural images normally have compressible coefficients,

\footnotetext{
* Correspondence: mai.xu06@imperial.ac.uk

* Correspondence: mai.xu06@imperial.ac.uk Republic of China
}

decaying rapidly enough to zero when sorted, and thus can be approximated well as $S$-sparse.

\section{A Compressive sensing}

Most recently compressive sensing (CS), as a sampling method in image compression, has been proposed [5-7], in order to compress the sparse/compressible signals $\boldsymbol{x}$ directly into acquisition during the sensing procedure. In CS, given $M \times N$ random measurement matrix $\boldsymbol{\Phi}$, we are able to achieve $M$-dimensional measurement values $y$ via inner product:

$$
y=\boldsymbol{\Phi} x
$$

where each entry $y_{s}$ of $\boldsymbol{y}$ represents the value measured by measurement vector $\varphi_{s}$ that is $s$ th row of $\boldsymbol{\Phi}$. It has been proved [6] that image can be robustly recovered from $M=O(S \log (N / S))$ measurements. In practice, $M=4 S$ measurements are required for precise recovery as reported in [4], and therefore, compression can be reached by sensing and storing $M$ measurements $y$. It has been proved that the signal can be recovered by seeking the sparest $\boldsymbol{x}$ with the solution of convex program [8]:

$$
\hat{\boldsymbol{x}}=\underset{x^{\prime}}{\arg \min }\left\|\boldsymbol{x}^{\prime}\right\|_{1} \quad \text { s.t. } \boldsymbol{y}=\boldsymbol{\Phi} \boldsymbol{x}^{\prime}
$$

Equation 2 can be solved by a linear program within polynomial time [9]. For reducing the computational time, some other approaches have been proposed in the 
spirit of either greedy algorithms or combinatorial algorithms.

These include orthogonal matching pursuit (OMP) [10], StOMP [11], subspace pursuit [12] and CoSaMP [13].

It is attractive that CS is also applicable to images with sparse or compressible coefficients in the transform domain since $\boldsymbol{y}$ can be written as $\boldsymbol{y}=\boldsymbol{\Phi} \boldsymbol{\Psi} \boldsymbol{x}$, in which $\boldsymbol{\Phi} \boldsymbol{\Psi}$ can be seen as $M \times N$ measurement matrix. In the sequel, without generality loss we shall focus on the images, sparse or compressible in the pixel domain. However, in our experiments of Section 4, we shall also consider the images, compressible in wavelet domain.

\section{B Basic idea}

Beyond CS, most recently, various extensions of CS have been proposed. CS, at heart, utilizes the prior knowledge of the sparsity of signal to compress the signal. Actually, some signals, such as digital images, have some prior knowledge other than sparsity. For example, we know that the nonzero coefficients of images usually cluster together, and a model-based CS was thus proposed in [14-16] to integrate the prior knowledge of signal structure in CS for reducing the amount of measurements required for the recovery of images. However, to our best knowledge, all the state-of-the-art model-based CS approaches only concentrate on the prior knowledge of the locations of nonzero value pixels in digital images and assume that all the $N$ pixel values of a digital image $\in \mathbb{R}^{N}$. For example, [17] proposed $(S, C)$-model-based CS for reconstructing the $S$-sparse signal with the prior knowledge of block sparsity model in which there are at most $C$ clusters with respect to the locations of nonzero coefficients of the signal. This approach is applicable to some practical problems such as MIMO channel equalization. However, in some other applications, the values of the sparse signal rather than nonzero-valued locations cluster together. Therefore, in this paper, we consider sparse signals with the prior knowledge of $K$-clustervalued coefficients, either in the canonical (pixel) domain or in the wavelet domain.

As a matter of fact, it has been shown [18] that for the most digital images, the intensities of each pixel are usually the subspace $^{\mathrm{a}}$ of $[0,255]$. The motivation of this paper is thereby to extend the model-based CS theory to include such prior knowledge. Then, we propose a reconstruction approach based on $K$-cluster-valued intensities for CS (called $K$-cluster-valued CS) to incorporate $K$-means algorithm in CS for recovering the images using only $K$ clusters of nonzero intensity values, $\left\{\mu_{1}, \mu_{2}, \ldots, \mu_{K}\right\} \subseteq[1,255]$. Once the measurement number $M$ is less than required $4 S$ for image compression with CS, there may exist several unreasonable solutions to the estimation of target image. However, during the reconstruction procedure, the proposed $K$-cluster-valued CS avoids the possibility of intensity values being assigned beyond $K$ clusters: $\left\{\mu_{1}, \mu_{2}, \ldots, \mu_{K}\right\}$, and it thus may be capable of discarding those unreasonable solutions. So, $K$-cluster-valued CS is possible to reduce the number of measurements $M$ required for robust image recovery. Note that in a gray image even we set cluster number $K$ to be 255 at limit, $K$-cluster-valued CS can still avoid the recovered intensity values of each pixel to be greater than 255 or less than 0 in conventional CS. Since our proposed $K$-cluster-valued CS is an extension of model-based CS, we shall briefly review the modelbased CS in the following section.

For instance, when we apply CS in compressing the binary image, the measurement number $M$ may be reduced with the prior knowledge that only one cluster of nonzero intensity values is available for reconstructing image. As illustrated in Figure 1, CS recovery algorithm, even with insufficient measurements, is able to refuse the solution not supported by the prior knowledge of only binary values being available for image intensities. Consequently, $K$-cluster-valued CS is possible to reduce the measurement number for precise reconstruction of the target image. Since our proposed $K$ cluster-valued CS is an extension of model-based CS, we shall briefly review the model-based CS in the following section.

\section{Overview of model-based CS}

Model-based CS [14] incorporates some other prior knowledge rather than the sparsity or compressibility of signal in CS. It is intuitive that the restriction of such an additive prior knowledge may decrease the redundancy of measurements in CS, and the reduction in measurement number $M$ of CS may therefore be possible.

In order to introduce the model-based CS, let us first consider the model-based restricted isometry property (RIP). Here, we define structured $S$ sparsity model $\mathcal{M}_{S}$ as the union of $m_{S}$ subspaces subjective to $\|\boldsymbol{x}\|_{0} \leq S$. Thus, the prior knowledge of the $S$-sparse signals can be encoded in $\mathcal{M}_{\mathcal{S}}$. Then, RIP of [19] can be rewritten as

- An $M \times N$ matrix $\boldsymbol{\Phi}$ has the $\mathcal{M}_{S}$-restricted isometry property with constant $\delta_{\mathcal{M}_{S}}$ for all $\boldsymbol{x} \in \mathcal{M}_{S}$, we have

$$
\left(1-\delta_{\mathcal{M}_{s}}\right)\|\boldsymbol{x}\|_{2}^{2} \leq\|\boldsymbol{\Phi} \boldsymbol{x}\|_{2}^{2} \leq\left(1+\delta_{\mathcal{M}_{s}}\right)\|\boldsymbol{x}\|_{2}^{2}
$$

It has been proved [19] that if

$$
M \geq \frac{2}{c \delta_{\mathcal{M}_{S}}^{2}}\left(\ln \left(2 m_{S}\right)+S \ln \frac{12}{\delta_{\mathcal{M}_{S}}}+t\right)
$$

where $c$ is a positive constant, then $\Phi$ has $\mathcal{M}_{S}$-restricted isometry property with the probability at least $1-\mathrm{e}^{-t}$ given constant $\delta_{\mathcal{M}_{s}}$. It can be seen from Equation 4 that as number of $m_{S}$ increases, more 


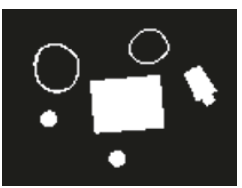

(a)

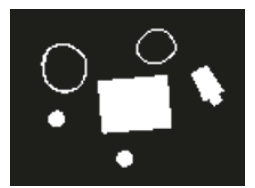

(b)

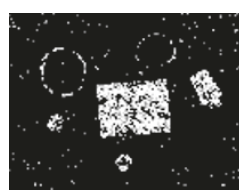

(c)

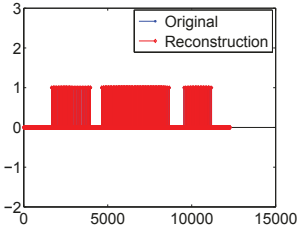

(d)

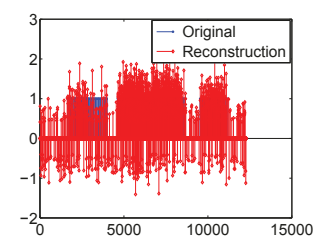

(e)

Figure 1 (a) The original $96 \times 128$ binary image, (b) the reconstructed image using CS with the prior knowledge that only one cluster of nonzero intensity values exist, and (c) the reconstructed image without any prior knowledge. (d) The reconstruction results of 1cluster-intensity-based CS, after aligning $96 \times 128$ pixels of the image to be a vector with 12,288 entries, and (e) the reconstructing results of conventional CS, after aligning $96 \times 128$ pixels of the image to be a vector with 12,288 entries. The sparsity $S$ in this image is 1,592 , and measurement number $M$ here is $3 S$, which is smaller than 45 [4] required for the accurate recovery of image in conventional CS.

measurement numbers will be required for recovering the target signal, and model-based CS increasingly resembles conventional $\mathrm{CS}$, becoming equivalent to conventional $C S$ at limit $m_{S}$ of being $\left(\begin{array}{c}N \\ S\end{array}\right)$. It satisfies the intuition that the more prior knowledge we have (such that $m_{S}$ decreases), the less measurement number is required for target signal recovery.

Next, we define $M(x, S)$ as the algorithm obtaining the best $S$-sparse approximation of $\boldsymbol{x}, \hat{x}$ :

$$
M(x, S)=\underset{\hat{x} \in \mathcal{M}_{S}}{\arg \min }\|\boldsymbol{x}-\hat{\boldsymbol{x}}\|_{2}
$$

Then, the prior knowledge can be encoded in algorithm $M$ in advance. Given such an algorithm, the recovery method CoSaMP [13] may be extended for model-based CS (See Algorithm 1 of [14] for the summary of model-based CoSaMP). Also, note that there is no difference between conventional CS and modelbased CS in the measuring/sampling step summarized in Equation 1.

It has also been proved in [14] that error bound of model-based CoSaMP is

$$
\left\|\boldsymbol{x}-\hat{\boldsymbol{x}}^{i}\right\|_{2}=2^{-i}\|\boldsymbol{x}\|_{2}+15\|\boldsymbol{\varepsilon}\|_{2}
$$

for $\mathcal{M}_{S}$-RIP constant $\delta_{\mathcal{M}_{4 S}} \leq 0.1$. In Equation $6, \varepsilon$ is the noises additive to the measurements, $i$ is the iteration number and $\hat{x}^{i}$ is the estimated $\hat{x}$ at the $i$ th iteration. This equation guarantees the error of model-based CS to be the same as conventional CS.
In a word, on the basis of prior knowledge encoded in advance, the model-based CS is capable of reducing the measurement numbers without increasing any error bound.

\section{K-cluster-valued CS}

In this section, we provide the detail of the proposed approach on the basis of model-based CS. It is intuitive that a digital image is comprised of the pixels with several clusters (at most 256) of the intensities, rather than all possible values used for estimating the image/signal in conventional CS or model-based CS [14]. So, structured $S$ sparsity model $\mathcal{M}_{S}$ as mentioned in Section II is set here to be $\|\hat{\boldsymbol{x}}\|_{0} \leq S$ s.t. $\hat{x}_{n} \in\{0,1,2, \ldots, 255\}$ for image reconstruction. Then, the algorithm of Equation 5 for obtaining $\hat{\boldsymbol{x}}=\left\{\hat{x}_{n}\right\}_{1}^{N}$ is

$$
M(x, S)=\underset{\hat{x}_{n} \in\{0,1,2, \ldots, 255\}}{\arg \min } \sum_{n=1}^{N}\left\|x_{n}-\hat{x}_{n}\right\|_{2}, \quad \text { s.t. } \quad\|\hat{x}\|_{0} \leq S
$$

The $K$-means algorithm [20] ensures that the clusters of data with the same or similar values can be identified in the same data set. So, $K$-means algorithm can be applied to the algorithm of Equation 7 using at most $K$ $=255$ clusters of nonzero intensities to reconstruct the target image at each iteration of recovery algorithm in CS. However, in practice, since most digital images have less than 255 clusters of nonzero intensities (the statistical analysis will be presented in the last part of this section), $K$ is normally set to be less than 255 for image reconstruction. Even though $K$ is the rough estimation 
of real cluster numbers, $K$-means algorithm still works in model-based CS due to the fact that the goal of $K$ means algorithm is to minimize $\sum_{n}\left\|x_{n}-\hat{x}_{n}\right\|$, where $\hat{x}_{n} \in\left\{\mu_{1}, \mu_{2}, \ldots, \mu_{K}\right\}$ with $\mu_{k}$ being the center of $k$ th cluster.

As assumed above, $\hat{x}^{i}=\left\{\hat{x}_{1}^{i}, \hat{x}_{2}^{i}, \ldots, \hat{x}_{N}^{i}\right\}$ are the estimated gray values of all pixels in the image at the $i$ th iteration of recovery algorithm for CS. Then, we have the prior knowledge that all the nonzero values of $\hat{x}^{i}$ can be replaced at iteration $i$ by $K$ clusters of intensity values $\left\{\mu_{1}^{i}, \mu_{2}^{i}, \ldots, \mu_{K}^{i}\right\}$, in which each $\mu_{k}^{i} \in[1,255]$. Our aim then is to partition the nonzero values of $\left\{\hat{x}_{1}^{i}, \hat{x}_{2}^{i}, \ldots, \hat{x}_{N}^{i}\right\}$ into $K(\leq 255)$ clusters $^{\mathrm{b}}$, at each iteration of CS reconstruction step. To this end, we may apply $K$ means algorithm [20] in model-based CS for target image recovery.

Given each nonzero $\hat{x}_{n}^{i}$ in $\hat{x}^{i}$, there is a corresponding set of binary indicator $r_{n k}^{i} \in\{0,1\}$, showing which of $K$ clusters the intensity value of $n$th pixel $\hat{x}_{n}^{i}$ is assigned to. If $\hat{x}_{n}^{i}$ is assigned to be $\mu_{k}^{i}$, then $r_{n k}^{i}=1$, and $r_{n j}^{i}=0$ for $j \neq$ $k$. Next, in $K$-cluster-valued CS, we may obtain $r_{n k}^{i}$ and $\mu_{k}^{i}$ at iteration $i$ by minimizing the objective function:

$$
J=\sum_{n=1}^{N} \sum_{k=1}^{K} r_{n k}^{i}\left\|\hat{x}_{n}^{i}-\mu_{k}^{i}\right\|_{2}
$$

For the purpose of minimizing $J$, we may apply an iterative procedure involving two successive optimizations. The first optimization deals with minimizing $J$ with respect to $r_{n k}^{i}$, keeping $\mu_{k}^{i}$ fixed. Then, the second optimization involves minimizing $J$ relating to $\mu_{k}^{i}$, with $r_{n k}^{i}$ being fixed. Therefore, at iteration $i$, there are twostage optimizations for updating $r_{n k}^{i}$ and $\mu_{k}^{i}$, respectively:

1. Maximization: Since Equation 8 is a linear function of $r_{n k}$, this optimization can be easily solved by setting $r_{n k}$ to be 1 once $k$ makes $\left\|\hat{x}_{n}^{i}-\mu_{k}^{i}\right\|_{2}$ minimum. In another word, each nonzero $\hat{x}_{n}^{i}$ is assigned to the closest $\mu_{k}^{i}$. So, this may be represented as

$$
r_{n k}^{i}= \begin{cases}1 & \text { if } k=\arg \min _{j}\left\|\hat{x}_{n}^{i}-\mu_{k}^{i}\right\|_{2} \text { and } \hat{x}_{n}^{i} \neq 0 \\ 0 & \text { otherwise }\end{cases}
$$

2. Expectation: In this stage, $r_{n k}$ has been fixed such that Equation 8 can be minimized with respect to $\mu_{k}^{i}$ by setting its derivative to be 0 :

$$
2 \sum_{n=1}^{N} r_{n k}^{i}\left(\hat{x}_{n}^{i}-\mu_{k}^{i}\right)=0
$$

So, Equation 8 can be solved by

$$
\mu_{k}^{i}=\frac{\sum_{n=1}^{N} r_{n k}^{i} \hat{x}_{n}^{i}}{\sum_{n=1}^{N} r_{n k}^{i}}
$$

The above two stages are then repeated until reaching at convergence. However, it may converge to a local minimization rather than global minimization. Therefore, a good initialization procedure can reduce the oscillations and improve the performance of the proposed approach. Fortunately, we have the prior knowledge that for an image, $\left\{\mu_{1}^{i}, \mu_{2}^{i}, \ldots, \mu_{K}^{i}\right\} \subseteq\{1,2, \ldots, 255\}$. Hence, in the proposed $K$-cluster-valued CS, the initial values of $\mu_{k}^{i}$ may be chosen randomly from $[1,255]$. Then, the iterations of the two stages of $K$-means are run until there is trivial change in objective function $J$ in Equation 8 or until some maximum number of iterations (100 as set in Section 4) is exceeded.

After these iterative two stages, the values of each $\hat{x}_{n}^{i}$ are set to be $\mu_{k}^{i}$ if $r_{n k}=1$. The proposed $K$-clustervalued CoSaMP recovery algorithm for CS is summarized in Table 1 . Note that the measuring method of $K$ cluster-valued CS is same as the conventional CS, which has already been expressed in Equation 1 of Section 1.

At each iteration, not only the measurement residual but also EM is applied for estimating the target signal. Since there are only a few clusters of intensity values for the target image, it is able to reduce the error caused by assigning unreasonable estimated values (e.g., more than 255 clusters) to each pixel at each iteration. Although the estimation error of the image may influence the clustering accuracy at each iteration, the minimization of Equation 8 also makes such influence minimal and the proposed approach converges after a few iterations. The computational time reported in section 4 also reveals that the robust of clustering.

Then, we have to answer how to confirm cluster number $K$ for $K$-cluster-valued CS. Here, we first consider the images sparse in pixel domain, and we statistically tested on 1,000 images from Caltech 101 and Berkeley Segmentation Database to obtain the optimal cluster numbers of pixel intensities for $K$-means in each image, which make the PSNR greater than $20 \mathrm{~dB}$ (since the acceptable value for the quality of lossy image compression is above $20 \mathrm{~dB}$ [21]). The statistical results are shown in Figure 2. As seen from this figure, more than $85 \%$ cluster numbers of intensities range below 10 , and thus 10 may be chosen as an optimal cluster number for images sparse in pixel domain.

However, the images are hardly sparse in pixel domain. Therefore, we need to consider clustering wavelet coefficients of images. Toward the optimal cluster 


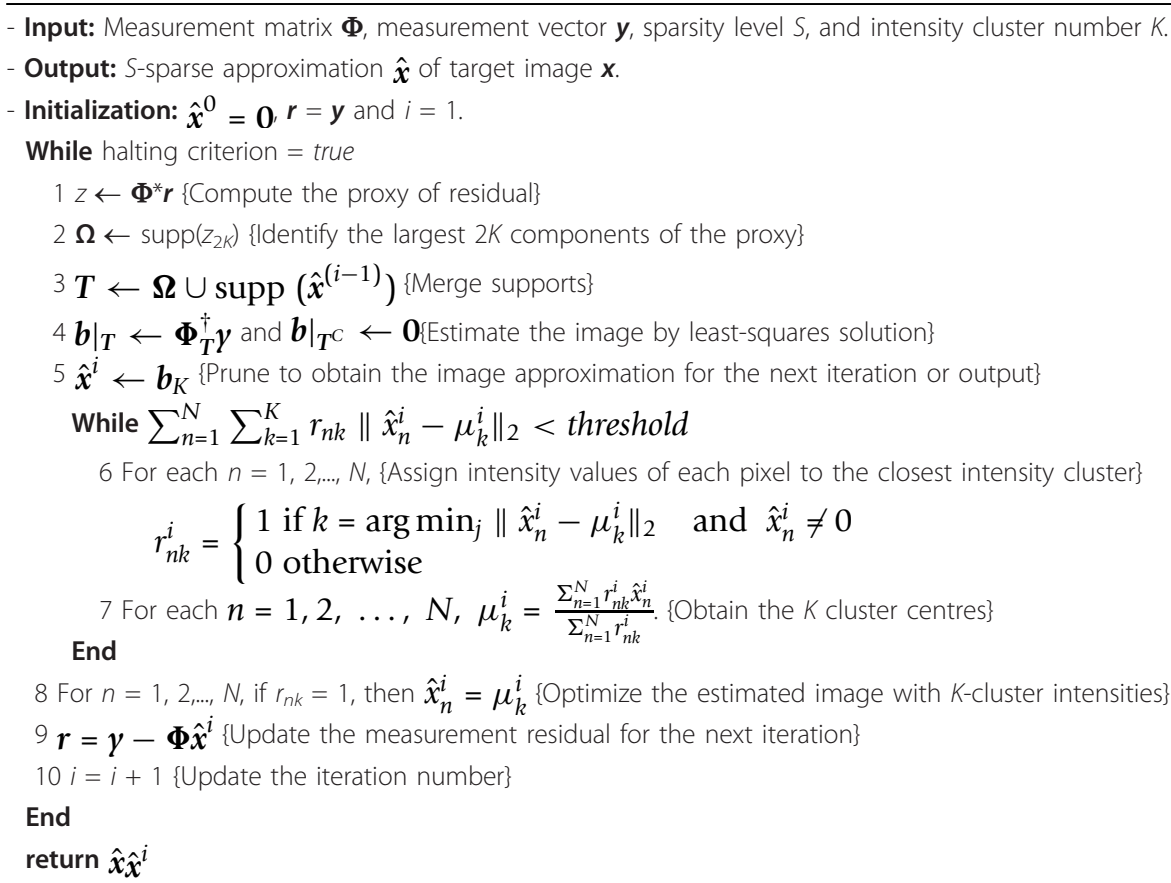

number of wavelet coefficients, we also statistically tested on the same 1,000 images as above with the following procedure: (1) obtain the wavelet coefficients of each image, and set wavelet coefficients less than 20 to be 0 ; (2) reconstruct the image with nonzero wavelet coefficients by non- $K$ means algorithm and compute the PSNR of reconstructed image; (3) reconstruct the image by $K$ means algorithm with increasing number of clusters of nonzero wavelet coefficients, and once the cluster number makes the difference in the PSNRs between images reconstructed by non- $K$ means and $K$-means algorithms less than $2 \mathrm{~dB}$, we output this cluster number as optimal one. The statistical results are then

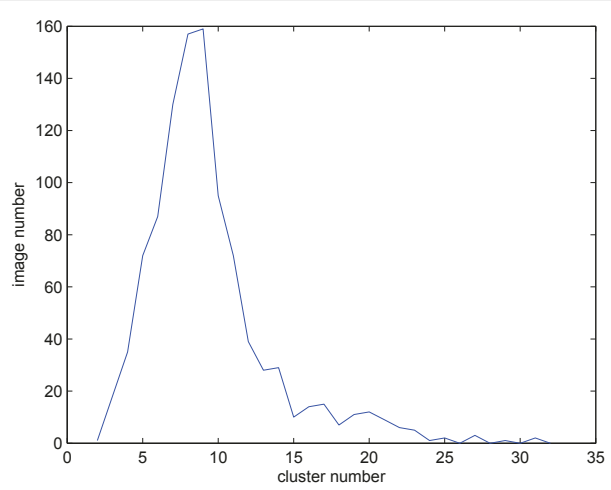

Figure 2 The number of images along with their optimal cluster numbers of intensities for $\boldsymbol{K}$-mean algorithm. Note that the cluster number is chosen to be optimal one once it makes the accuracy of $K$-mean algorithm greater than $20 \mathrm{~dB}$. demonstrated in Figure 3. From this figure, we may confirm that clustering is a reliable prior in the wavelet transform for images, and 40 is an optimal cluster number for wavelet coefficients.

\section{Experimental results}

In this section, experiments were performed for validating the proposed $K$-cluster-valued CS. For comparison, we also applied the conventional CS to exactly the same images. In all the experiments, we utilized random Gaussian matrix as the measurement matrix $\boldsymbol{\Phi}$ on the images. For conventional CS and $K$-cluster-valued CS, the maximum iteration numbers of CoSaMP were both set to be 30 . Besides, the iterations can also be halted

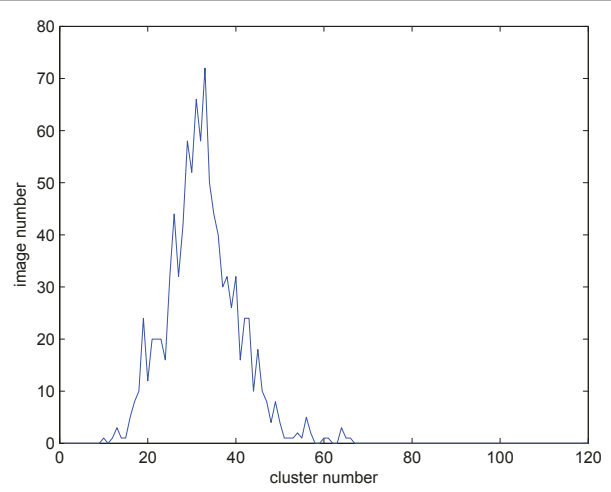

Figure 3 The number of images along with their optimal cluster numbers of wavelet coefficients for $K$-mean algorithm. 
once $\left\|\hat{\boldsymbol{x}}^{i}-\hat{\boldsymbol{x}}^{i-1}\right\|_{2}<10^{-2}\left\|\hat{\boldsymbol{x}}^{i}\right\|_{2}$. For $K$-cluster-valued $\mathrm{CS}$, the iterative two stages of $K$-means algorithm are repeated 100 times. The experiments have been performed under the following system environments: Matlab R2008b on a computer with Pentium(R) D 2.8GHz CPU and 3-GB RAM. Section A focuses on utilizing the $K$-cluster-valued and conventional CSs to compress one lunar image relying on a canonical (pixel) sparsity basis. This subsection shows the results in detail. In Section B, we demonstrate the experiments on other extensive images in brief. This subsection mainly concentrates on the 2D images, using either a canonical sparsity basis or wavelet sparsity basis, as the input to our experiments. In addition, the experiments on some background subtracted images in color are demonstrated as well.

\section{A One experiment in detail}

First of all, a lunar image (Figure 4a) was tested on conventional $C S$. Then, the reconstructed image is shown in Figure $4 \mathrm{~b}$ using the recovery methods of CoSaMP, with $M=3 S=5217$ random Gaussian measurements, where $S=1739$ indicates the nonzero intensity values of the lunar image. Note that the measurement number $M$ is approximately $\frac{1}{3} N$, which is high compared to the undersampling ratio in wavelet domain. However, the advantage of the proposed and conventional CSs is that the image can be compressed directly during the acquisition procedure. Further, we utilized the $K$-clustervalued CS to reconstruct the target lunar image given the same measurements. The recovery results are shown in Figure 4c-f with cluster numbers $K=2,5,10$ and 50, respectively. From Figure 4, it can be seen that when the measurements are insufficient (less than 4S), K-cluster-valued CS outperforms over conventional CS referring to recovery accuracy. We may see that once the cluster number increases, the reconstructed images become smoother and the accuracy thus will be better. Moreover, there is almost no difference between the images reconstructed by 10 -cluster and 50 -cluster, and it agrees with statistical analysis of the cluster number introduced in the above section.

From the viewpoint of computational time, as can been seen from Figure 4, K-cluster-valued CS runs faster than conventional CS when cluster number $K$ is small (e.g., $K=2,5$ and 10 ). It may be due to the fast convergence of $K$-cluster-valued CoSaMP caused by more accurate recovery result at each iteration.

Next, we shall compare the recovery error of conventional and $K$-cluster-valued CSs in terms of peak signalto-noise ratio (PSNR). PSNR refers to the ratio between the maximum possible power of a signal and the power of corrupting noise that affects the fidelity of its representation. Since many signals have very wide dynamic ranges, PSNR is normally expressed in terms of the logarithmic decibel scale. In our experiments, PSNR, as a measure of quality of lossy image compression, is defined by:

$$
\operatorname{PSNR}=20 \log _{10} \frac{255 \sqrt{N}}{\|x-\hat{x}\|_{2}}
$$

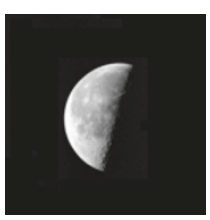

(a) Original image

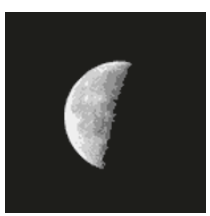

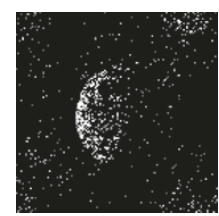

(b) Conventional CoSaMP

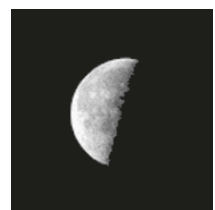

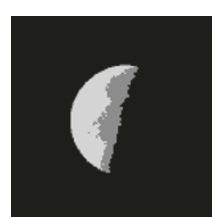

(c) 2-clusters intensities based CoSaMP

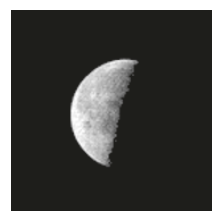

(d) 5-clusters intensities based CoSaMP (e) 10-clusters intensities based CoSaMP (f) 50-clusters intensities based CoSaMP

Figure 4 (a) The original lunar gray image (resolution: $128 \times 128$ ) with $S=1,739$ nonzero values. The random Gaussian measurement number of CS over this image is $M=3 S=5,217$ measurements, which is $\frac{5217}{128 \times 128} N\left(\sim \frac{1}{3} N\right)$. (b) The reconstructed image using conventional CoSaMP recovery method. (c)-(f) The images reconstructed with 2, 5, 10 and 50 clusters of values for intensities CoSaMPs. The computational time is (b) $5.82 \mathrm{~s}$ (c) $2.94 \mathrm{~s}$ (d) $3.24 \mathrm{~s}$ (e) $5.74 \mathrm{~s}$ and (f) $10.36 \mathrm{~s}$. 
where $N$ is the number of pixels at each image and 255 is the dynamic range of intensities of the image. $\boldsymbol{x}$ and $\hat{x}$ are the intensities of the original image and compressed image, respectively.

Then, we run Monte Carlo (50 times) simulation on computing the PSNR of image reconstruction via conventional and $K$-cluster-valued CSs. The results can be seen in Figure 5. Figure 5a shows the impact of measurement number $M$ on the performance of $K$-clustervalued and conventional CSs for the target image displayed in Figure 4. Since the acceptable value for the quality of lossy image compression is above $20 \mathrm{~dB}$ [21], Figure 5 a reveals that the proposed $K$-cluster-valued CS approach reaches at tolerable recovery result when $M=$ $3 S$, while the conventional CS fails ${ }^{\mathrm{c}}$. Also, it can be further seen that even when the measurement number is sufficient $(M=4 S)$, the performance of $K$-clustervalued $C S$ is superior to conventional one. Figure $5 \mathrm{~b}$ further shows the performance of $K$-cluster-valued CS given different cluster number $K$. We can observe that $K$-cluster-valued CS works well with different cluster numbers and that the more cluster number we choose, the better $K$-cluster-valued CS will perform in most cases.

\section{B More experiments in general}

In this subsection, we evaluated our proposed approach on three different images sets: (1) the image set chosen from Caltech 101 database contains five images, sparse in pixel domain; (2) the natural image set contains four images, sparse or compressible in wavelet domain; (3) background subtracted color image set.

For the first image set, since we have concluded in the above section that 10 can be seen as an optimal cluster number of intensities for the images sparse in pixel domain, we set $K$ of $K$-cluster-valued CS to be 10 . In addition, all the measurement numbers used for compressing these images were chosen to be $3 S$, which is less than the least measurement number $4 S$ required for successful reconstruction in conventional CS [4]. Then, the reconstruction results are presented in Figure 6, and these results show the better performance of $K$-clustervalued CS for compressing images that are sparse in standard domain.

With the second image set, we have evaluated the promise of the proposed CS approach on compressing the images in wavelet domain. Here, as aforementioned in Section 3, we chose the cluster number of wavelet coefficients for $K$-cluster-valued CS to be 40 as concluded in the above section. In order to obtain the more accurate results, the measurement numbers here were all set to be $3.5 S$, where $S$ is the number of largest $S$ wavelet coefficients used for image reconstruction. Then, the input and output images are shown in Figure 7, and the PSNRs of the reconstructed images in this figure are further demonstrated in Table 2. Again, $K$-cluster-valued CS offers the better performance in compressing images, sparse in wavelet domain.

The $K$-clusters-valued CS is also applicable to background subtracted images. Here, we tested the proposed $K$-clusters-valued CS and conventional CS on the third image set with two background subtraction images from [16]. According to [16], these images are obtained by selecting at random two frames of video sequence and subtracting them in a pixel-wise fashion. For each image, we set the cluster number $K$ to be 10 as well. Then, we performed $K$-clusters-valued CoSaMP and conventional CoSaMP under $M=3 S$ Gaussian random

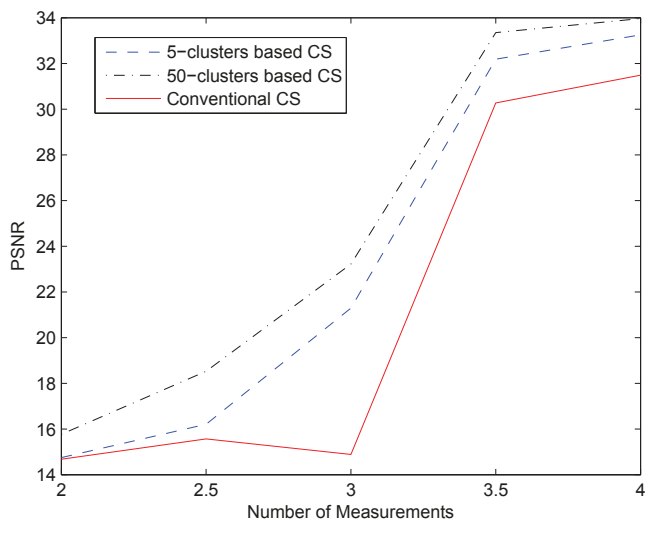

(a)

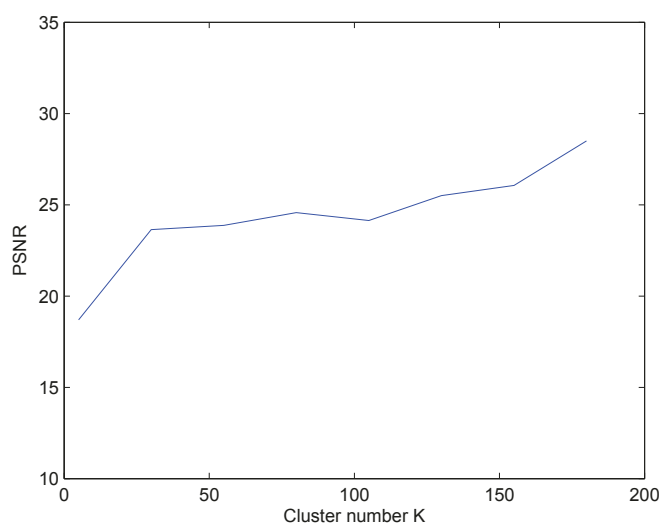

(b)

Figure 5 (a) The reconstruction error of Figure 4 measured by PSNR. In this figure, the PSNR (vertical axis) is shown along with various measurement number and the values at the horizontal axis indicate how many times of sparsity level $S$. (b) The results of PSNR output by Kcluster-valued CS with various cluster numbers $K$. The measurement number $M$ here is set to be $3 S$. 

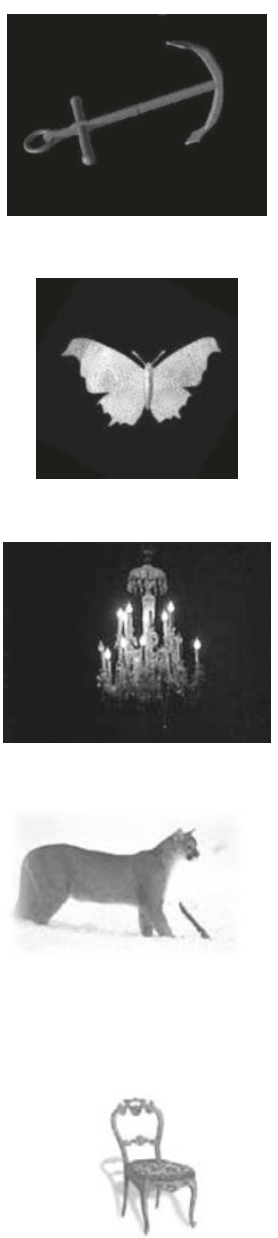

(a) Original image
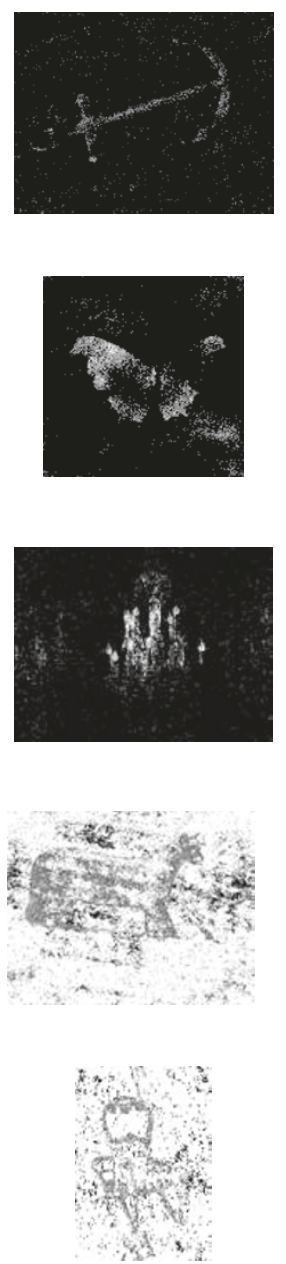

(b) Conventional CoSaMP
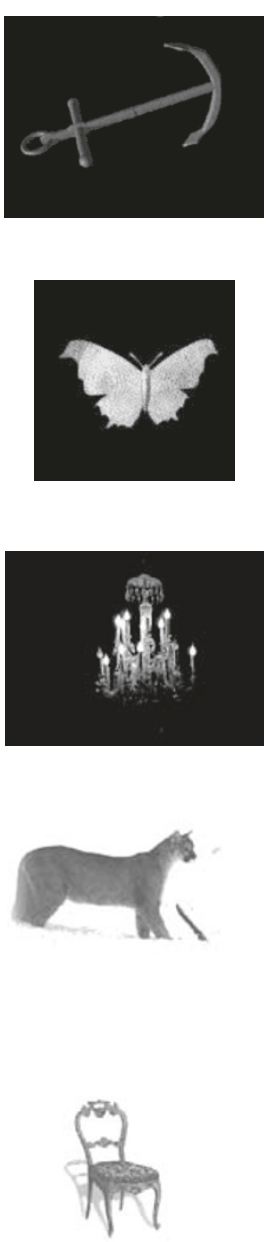

(c) 10-cluster intensities valued

\section{CoSaMP}

Figure 6 (a) The original gray images. The measurement numbers of both conventional and K-cluster-valued CS over these images are $M=$ $3 S$, where $S$ is the sparse level. (b) The reconstructed images using conventional CoSaMP recovery method. (c) The images reconstructed by $K$ cluster-valued CoSaMP. Note that cluster number $K$ was chosen to be 10 for all these images.

measurements. The recovery results are shown in Figure 8. We may see from this figure that $K$-cluster-valued CS outperforms conventional one and that $K$-cluster-valued CS is also capable of recovering background subtracted images with insufficient measurements (e.g., $3 S$ measurements).

\section{Conclusions}

In this paper, in order to compress the image, we have aimed to propose an advanced model-based CS, named $K$-cluster-valued CS, which utilizes $K$-means algorithm as the model for CS. In contrast to conventional CS, the proposed $K$-cluster-valued CS incorporates the prior knowledge that only $K$ clusters values of intensities are available for all the pixels of an image. In this paper, we also investigated cluster number $K$ as prior knowledge. Such prior knowledge goes beyond the simple sparsity/ compressibility of CS and therefore has the advantage in using fewer measurements than conventional CS for accurate image reconstruction. This way, $K$-clustervalued CS is applicable to other $K$-cluster-valued signals (e.g., binary digital signals) besides the images. Also, it is applicable to other model-based CS by considering all the prior knowledge together. Moreover, the experiments were performed and presented to validate the proposed approach. 

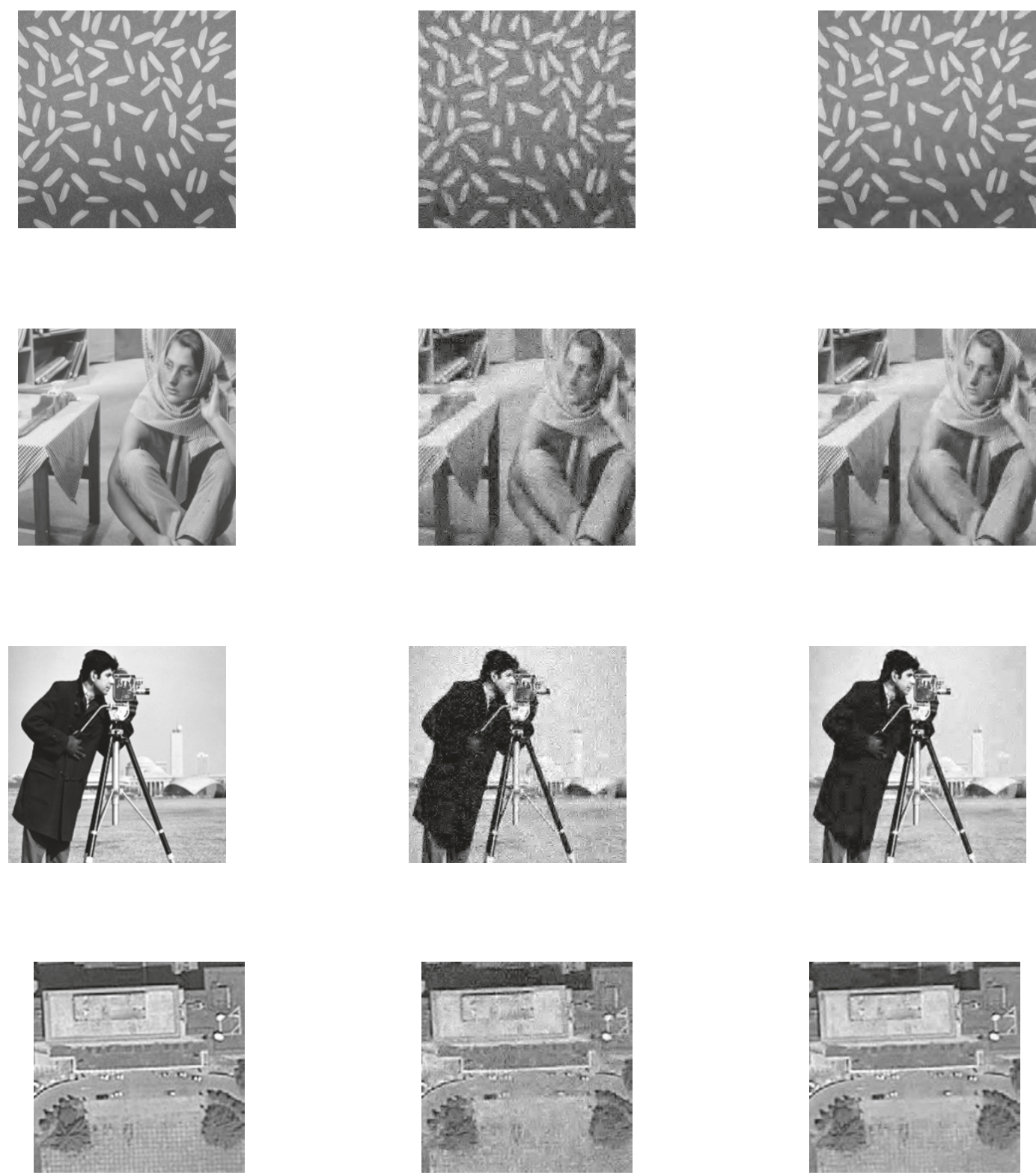
(a) Original image

(b) Conventional CoSaMP

(c) K-cluster valued CoSaMP

Figure 7 (a) The original gray images measured by CS with wavelet sparsity basis. The measurement numbers of both conventional and K-cluster-valued CS over these images are $M=3.55$, where $S$ is the number of compressible wavelet coefficients greater than threshold 20. (b) The reconstructed images using the conventional CoSaMP recovery method. (c) The images reconstructed by K-cluster-valued (wavelet coefficients) CoSaMP. Note that cluster number $K$ was chosen to be 40 for all these images.

Table 2 The PSNRs of reconstructed images of Figure 7

\begin{tabular}{ccccc}
\hline Methods & (a) & (b) & (c) & (d) \\
\hline Conventional CoSaMP & 22.61 & 22.10 & 20.85 & 21.67 \\
K-cluster-valued CoSaMP & 31.03 & 27.39 & 28.20 & 26.95 \\
\hline
\end{tabular}

\section{Endnotes}

${ }^{\text {a }}$ This is the usual case since an image is normally comprised of a few categories of objects with limited color intensities as exploited in computer vision community. ${ }^{\mathrm{b}}$ The $K$ clusters can also be applied in color image by extending each gray value $\hat{x}_{n}^{i}$ to be 3 space comprising 

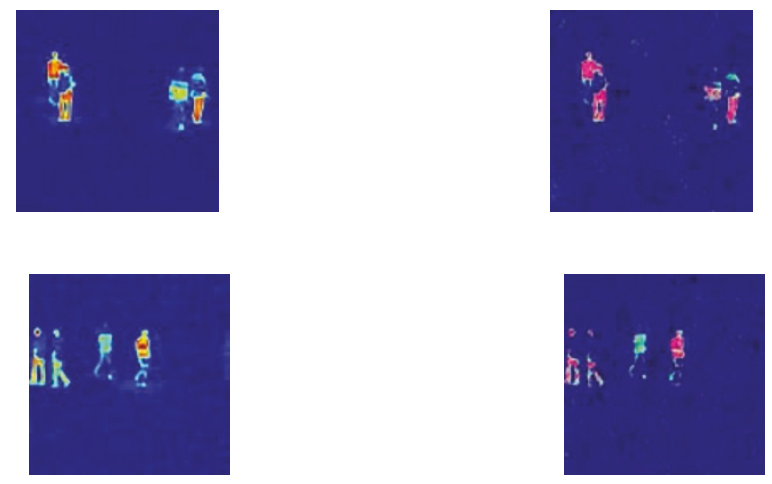

(b) Conventional CoSaMP

(a) Original image
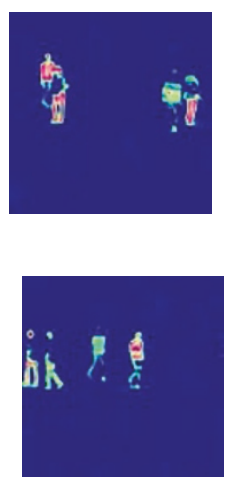

(c) 10-clusters intensities

valued CoSaMP

Figure 8 (a) Two original background subtraction images. The recovery results are shown in (b) for conventional CS and (c) for K-clustervalued $C S$, using $M=3 S$ random Gaussian measurements for each image. Note that cluster number $K$ was 10 for $K$-cluster-valued CS.

the intensities of the red, blue and green channels. ${ }^{\mathrm{c}}$ It is due to the fact that conventional CS does not have any prior knowledge of the range of intensity values of the pixels.

\section{Acknowledgements}

This work was partially supported by China National Basic Research Program (973) under Grant number 2007CB310600 and partially supported by NSFC 30971689.

\section{Competing interests}

The authors declare that they have no competing interests.

Received: 18 February 2011 Accepted: 26 September 2011 Published: 26 September 2011

\section{References}

1. M Petrou, C Petrou, Image Processing: The Fundamentals, 2nd edn. (Wiley, Amsterdam, 2010)

2. S Mallat, A Wavelet Tour of Signal Processing (Academic Press, New York, 1999)

3. D Taubman, M Marcellin, JPEG 2000: Image Compression Fundamentals, Standards and Practice, 1st edn. (Kluwer Academic, Dordrecht, 2001)

4. E Candès, M Wakin, An introduction to compressive sampling. IEEE Signal Process Mag. 25(2), 21-30 (2008)

5. E Candès, J Romberg, $T$ Tao, Robust uncertainty principles: exact signal reconstruction from highly incomplete frequency information. IEEE Trans Inf Theory. 52(02), 489-509 (2006)

6. E Candès, $T$ Tao, Near-optimal signal recovery from random projections: Universal encoding strategies?. IEEE Trans Inf Theory 52(12), 5406-5425 (2006)

7. D Donoho, Compressed sensing. IEEE Trans Inf Theory. 52(4), 1289-1306 (2006)

8. E Candès, J Romberg, T Tao, Stable signal recovery from incomplete and inaccurate measurements. Commun Pure Appl Math. 59(8), 1207-1223 (2006). doi:10.1002/cpa.20124

9. S Boyd, L Vandenberghe, Convex Optimization (Cambridge University Press, Cambridge, 2004)

10. J Tropp, A Gilbert, Signal recovery from random measurements via orthogonal matching pursuit. IEEE Trans Inf Theory. 53(12) 267-288 (2007)

11. DL Donoho, Y Tsaig, I Drori, J luc Starck, Sparse solution of underdetermined linear equations by stagewise orthogonal matching pursuit. Tech Rep, March, 1-39 (2006)
12. W Dai, O Milenkovic, Subspace pursuit for compressive sensing signal reconstruction. IEEE Trans Inf Theory 55(5), 267-288 (2009)

13. D Needell, J Tropp, Cosamp: iterative signal recovery from incomplete and inaccurate samples. Appl Comput Harmon Anal. 26(3), 301-321 (2009). doi:10.1016/j.acha.2008.07.002

14. R Baraniuk, V Cevher, M Duarte, C Hegde, Model-based compressive sensing. IEEE Trans Inf Theory 56(4), 1982-2001 (2010)

15. Y Eldar, $\mathrm{P}$ Kuppinger, $\mathrm{H}$ Bolcskei, Compressed sensing of block-sparse signals: uncertainty relations and efficient recovery. IEEE Trans Signal Process (2009)

16. V Cevher, M Duarte, C Hegde, R Baraniuk, Sparse signal recovery using markov random fields, in Proceedings of NIPS (2008)

17. V Cevher, P Indyk, H Chinmay, R Baraniuk, Recovery of clustered sparse signals from compressive measurements, in Proceedings of Sampta09 (2009)

18. CM Bishop, Pattern Recognition and Machine Learning, 1st edn. (Springer, New York, 2006)

19. T Blumensath, M Davies, Sampling theorems for signals from the union of finite-dimensional linear subspaces. IEEE Trans Inf Theory 55(4), 1872-1882 (2008)

20. SP Lloyd, Least squares quantization in pcm. IEEE Trans Inf Theory 28(2), 129-136 (1982). doi:10.1109/TIT.1982.1056489

21. N Thomos, N Boulgouris, M Strintzis, Optimized transmission of jpeg2000 streams over wireless channels. IEEE Trans Image Process 15(1), 54-67 (2006)

doi:10.1186/1687-6180-2011-75

Cite this article as: $\mathrm{Xu}$ and $\mathrm{Lu}$ : $K$-cluster-valued compressive sensing for imaging. EURASIP Journal on Advances in Signal Processing 2011 2011:75.

\section{Submit your manuscript to a SpringerOpen ${ }^{\mathcal{O}}$ journal and benefit from:}

- Convenient online submission

- Rigorous peer review

- Immediate publication on acceptance

- Open access: articles freely available online

- High visibility within the field

- Retaining the copyright to your article

Submit your next manuscript at $>$ springeropen.com 